\title{
Strategies for Prevention: Mental Health Nurse Burnout and Stress
}

\author{
Violeta Sadiku(Alterziu) \\ Lecturer at the Depertament of Psychology \\ Faculty of Educational Sciences \\ University of Elbasan "A.Xhuvani"
}

\begin{abstract}
The aim of this critical review was to identify prevention strategies that may be effective in the reduction of stress and burnout among mental health nurses. Diminished staff wellbeing, due to high levels of stress and burnout, has significant consequences at both the individual and the service level. Therefore, identifying effective prevention strategies may be beneficial in raising recruitment of mental health nurses, in prolonging retention, and may also have a positive impact on patient care. A search of the literature was undertaken utilising selected systematic review techniques, which identified seven articles as suitable for review. The results of the review found that three main prevention strategies were being utilised: clinical supervision, psychosocial intervention and social support. While all these strategies had the aim of minimising or preventing stress and/or burnout, they were all somewhat different in their focus and in their outcome measures. This factor, coupled with the paucity of high quality randomised intervention studies, makes it difficult to draw definitive conclusions concerning which intervention is most effective. The best currently available evidence suggests that prolonged clinical supervision is probably the best of the three options for the reduction of stress and burnout among mental health nurses, given the lack of high quality evidence and the magnitude and potential impact of this problem.
\end{abstract}

Keywords: Prevention, Stress, Burnout, Mental Health Nursing

\section{Introduction}

Employee stress and burnout commonly lead to myriad health-related problems that result in significant organizational consequences. Stress-related physical illnesses include heart disease, migraines, hypertension, irritable bowel syndrome, muscle, back and joint pain, duodenal ulcers, and mental health problems such as anxiety, depression, insomnia, and feelings of inadequacy, all of which can directly contribute toward absenteeism, decreased work performance, and ultimately, burnout. Nurses, en countering ongoing stress, are also more likely to eat poorly, smoke cigarettes, and abuse alcohol and drugs, all of which can lead to negative health conditions affecting personal well-being and subsequently, the quality and efficacy of patient care 1 .

The consequences of these conditions can have a significant impact on individual nurses and the ability to accomplish tasks; specifically, poor decision making, lack of concentration, apathy, decreased motivation, and anxiety may impair job performance, possibly resulting in lethal threats to patient safety. In addition, absenteeism due to stress-related problems requires the administrative use of unplanned and expensive replacement staff from agencies, or mandatory overtime for staff nurses, which further contributes toward an environment of stress and burnout. Interpersonal difficulties commonly stemming from stressful situations may compromise group cohesion, thus impacting the efficient functioning of the complex work units within the health care organization, and ultimately adding work to the already over-burdened middle and senior management teams.

When base staffing levels are low, due to high attrition rates, hospitals are forced to increase use of overtime or contract personnel. These labor costs are "forced costs" and generally are higher since they are of a staffing crisis intervention nature rather than a negotiated or strategically planned expense. With the current national situation of greater demand than

1 Barling, J. Drowning not waving: Burnout and mental health nursing. Contemporary Nurse, (2001) 11(2-3), $247-259$. 
supply of nurses, stress-related attrition simply adds greater labor costs to health care organizations, which are not recoverable from private or public insurance sources, and threatens safe patient care.

Low job satisfaction, controversial issues related to mandatory overtime, and poor staffing patterns create a ready culture for unionization. Consequently, additional costs to organizations will be incurred to deal with the union representatives, convene educational and public-hearing and community-relations related meetings, as well as manage the related distraction from the routine daily business of patient care. Union nurses are more willing to decline overtime and schedule changes to accommodate organizational demands resulting in less flexibility in staffing plans and more use of unplanned and expensive replacement staff from agencies. As such, interventions to prevent stress and burnout and retain nurses are critically necessary to ensure efficient and quality patient care and to potentially save hospital organizations billions of dollars annuall1.

\section{Clinical Supervision}

Clinical supervision is a formal process of professional support and learning (Edwards et al., 2006, p. 1008). Edwards et al. (2006) conducted a study to assess the effectiveness of clinical supervision involving Community Mental Health Nurses (CMHNs) working in eleven National Health Service (NHS) Trusts in Wales. The authors found that out of 260 respondents surveyed, 73 per cent $(n=189)$ had experienced six or more sessions of clinical supervision in their present position (p.1011). Significantly lower levels of emotional exhaustion and depersonalisation were reported among CMHNs who had received six sessions or more of clinical supervision. The authors of this study believe that this indicates a potential correlation between clinical supervision and a reduction in burnout rates. They also found that CMHNs who were younger, male and who had not experienced six or more sessions of clinical supervision showed higher depersonalisation scores ${ }^{2}$.

However, as the response rate was only 32 per cent of the CMHN's population within Wales, it is unlikely that the participants in this study were a representative sample. Another study by Hyrkas (2005), in which participants who had undergone a minimum of six months clinical supervision were surveyed, found similar results to Edwards et al. (2006) in terms of factors that influenced the effectiveness of the clinical supervision. Young, male nurses with a varied shift roster showed the least response to clinical supervision and least improvement in burnout scores (Edwards et al., 2006, p. 1012; Hyrkas, 2005, p. 540). This similarity in findings highlights a possible correlation between gender, age, the duration of clinical supervision, and its effectiveness, especially considering the fact that Hyrkas (2005) had an 85 per cent $(n=569)$ response rate across 14 sites, representing a wide geographical and professional spread (p. 540). A survey of mental health nurses, by White and Roche (2006) and the critical review by Edwards and Burnard (2003) also found that clinical supervision had a positive effect on the levels of stress and overall sense of well being experienced by mental health nurses. The three studies reviewed by Edwards and Burnard (2003) in their systematic review found that one year of clinical supervision significantly decreased the self-reported stress levels of mental health nurses. Furthermore, each of those studies had bias possibilities identified, limitations clearly defined and high response rates (average 82 per cent), thus supporting the findings of Edwards and Burnard (2003, p. 194). However, the survey conducted by White and Roche (2006) had a very low response rate of 21.2 per cent $(n=601)$, of which only one third had received clinical supervision for one year on a fortnightly or monthly basis, for between one and two hours per session. Nevertheless, of the respondents who did receive clinical supervision, 65 per cent self reported via questionnaire that they felt more developed professionally and personally, and hence felt less stressed (White \& Roche, 2006, p. 213).

\section{Psychosocial Interventions}

Psychosocial intervention (PSI) training aims to assist nurses to view patients' problems in a more empathetic way, whilst providing them with the skills required to intervene effectively in problematic situations (Edwards \& Burnard, 2003, p. 193). Some typical components of PSIs are relapse prevention, cognitive behavioural therapy and family interventions (Doyle,

1 Ewers, P., Bradshaw, T., McGovern, J., \& Ewers, B. Does training in psychosocial interventions reduce burnout rates in forensic nurses? (2002) Journal of Advanced Nursing, 37(5), 470-476.

2 Edwards, D., \& Burnard, P. A systematic review of stress and stress management interventions for mental health nurses. (2003) Journal of Advanced Nursing, 42(2), 169-200. 
Kelly, Clarke and Braynion, 2007, p. 16). The studies conducted by Doyle et al. (2007) and Ewers, Bradshaw, McGovern and Ewers (2002) examined the impact of PSI training on burnout rates in mental health nurses. Ewers et al. (2002) found that after completing the 20-day PSI training course there were significant positive changes, with the control group showing very little change (Ewers et al., 2002, p. 474). For example, in the intervention group, emotional exhaustion scores decreased by an average of three points and depersonalisation by four points, with personal achievement showing an average increase of four points when measured post-PSI training (Ewers et al., 2002, p. 475). Although these results indicated a correlation between PSI training and a reduction in burnout, the small sample size and potential bias, due to the principal researcher working in the secure unit, delivering most of the training and collecting the data, need to be taken into account when drawing conclusions from the results (Ewers et al., 2002, p. 475).

Doyle et al. (2007) conducted a similar study to Ewers et al. (2002), with participants representing all areas and disciplines being selected randomly and then allocated to either a control or training group. However, the same positive effects on burnout did not occur when PSI training was implemented, with no significant variation between the control and training groups in pre and post-testing (Doyle et al., 2007, p. 18). This difference in findings could have been due to the shorter intervention duration in the latter study, or that the Ewers et al. (2002) study sample incorporated both unqualified and qualified staff. Another possible reason is that Doyle et al. (2007) did not have the same representation of gender within each group that Ewers et al. (2002) did. Furthermore, both the studies used sample sizes of fewer than thirty participants, which is another reason to view these findings with caution.

\section{Social Support}

Jenkins and Elliot (2004) conducted a study in which a convenience sample of nursing staff from eleven acute adult mental health wards at four hospitals in London were assessed to identify the impact of social support on burnout. Social support was defined for the purpose of the study as support from co-workers, supervisors, spouse/partner, relatives and friends. The social support intervention involved substantial assistance and help with either a work task or emotional situation, such as listening sympathetically or the action of caring ${ }^{1}$. This study found a significant inverse correlation between co-worker support and $\mathrm{MBI}$ emotional exhaustion scores. Furthermore, support from supervisors was only half as effective in reducing emotional exhaustion as that from coworkers, with support from friends/relatives and spouse/ partner showing no significant change in emotional exhaustion scores. These significant differences in outcomes depending on who was delivering the support are notable. They could possibly be attributed to the fact that co-workers are more equipped to deal with workrelated problems than people who are more distant from the real life context that mental health nurses are experiencing, or who are (in the case of supervisors) generally less accessible (Jenkins \& Elliott, 2004, p. 628). However, due to the low response rate of 39 per cent, the use of convenience sampling and self-selection, the findings may not be representative and therefore no definitive statements can yet be made about the relationship between social support and the levels of burnout in mental health nurses (Jenkins \& Elliott, 2004, p. 628). In addition, the reviews by Edwards and Burnard (2003) found that social support programs offered no significant advantage in the reduction of burnout. Moreover, a test six months after the intervention found that emotional exhaustion scores had returned to pre-intervention levels, highlighting an obvious need for social support to be ongoing in order to maintain effectiveness (Edwards \& Burnard, 2003,p. 191).

\section{Discussion}

The research into strategies to reduce the stress and burnout being experienced by mental health nurses focuses on three main interventions: clinical supervision, psychosocial interventions and social support. The findings of the studies in this area are conflicting. This is in no small measure due to the fact that interventions, while fitting into broad categorical definitions, differ significantly in the nature of their specifics, their duration, and in the use of both comparison groups and pre and post-testing. The research, apart from a few exceptions, is also characterised by low response rates, so it is difficult to conclude definitively that any one of the interventions is more effective.

1 Tully, A. Stress, sources of stress and ways of coping among psychiatric nursing students (2004). Journal of Psychiatric \& Mental Health Nursing, 11(1), 43-7. 
However, some findings are suggestive. Of all three interventions, long duration clinical supervision currently has the best available evidence in support of its effectiveness. In order to establish better evidence for this, more studies need to be conducted to assess the exact length, delivery type and content of clinical supervision that is most beneficial. PSI training showed mixed results for reducing burnout. Therefore it would be reasonable to conclude at present that burnout in mental health nurses cannot be addressed effectively by implementing courses of this type until such time as further studies can be undertaken. The relationship between coworker support and reduced emotional exhaustion indicates that peer social (but not supervisor or family) support could also be influential in alleviating some of the effects of occupational stress, but again the lack of a body of research in this area makes it difficult to draw definitive conclusions about the effectiveness of this intervention.

All of the studies assessed for this review, except for Hyrkas (2005), used convenience sampling to select participants, thus raising the possibility of selection bias. This could operate in two ways. Participants could have chosen to participate as a way of making their feelings known or due to feeling particularly stressed or burnt out, which may have lead to inflated levels of reported stress or burnout. An alternative possibility is that the most stressed or burnt out nurses may have felt too exhausted and dissatisfied to participate, resulting in an underestimation of the real trends. Furthermore, the variance and lack of standardisation in sample sizes, duration of interventions and assessment tools used in the studies all contribute to problems with rigour and ability to generalize from the reported outcomes.

\section{Conclusion}

The most effective strategies for reducing the prevalence of stress and burnout in mental health nurses are yet to be identified. The complexity of the issue, with different clinical environments, different interventions and different outcome measures, makes it difficult to identify a single clearly effective strategy or set of strategies. Given these factors, and the relatively small volume of research in the area, it may be some time before this can be done. Further studies in the area need to address the identified areas of methodological deficiency, particularly lack of defined specificity for interventions and small non-randomly selected sample sizes.. Until these gaps in the evidence are addressed, the current best available evidence is that prolonged clinical supervision may be effective in alleviating the effects of stress and burnout for mental health nurses, and is the best option that we have at present for addressing this significant problem in mental health nursing practice.

\section{Bibliography}

Barling, J. (2001). Drowning not waving: Burnout and mental health nursing. Contemporary Nurse, 11(2-3), 247-259.

De Carlo, P. (2001). Mental health nursing: A stressful area of nursing practice? Paper presented at the Central Sydney Area Mental Health Service Winter Symposium. (Unpublished). Retrieved August 10, 2007, from http://www.cs.nsw.gov. au/mhealth/symposium/wintsym112001.htm

Edward, K., \& Hercelinskyj, G. (2007). Burnout in the caring nurse: Learning resilient behaviours. British Journal of Nursing, 16(4), 240-242.

Edwards, D., \& Burnard, P. (2003). A systematic review of stress and stress management interventions for mental health nurses. Journal of Advanced Nursing, 42(2), 169-200.

Edwards, D., Burnard, P., Hannigan, B., Cooper, L., Adams, J., Juggessur, T., et al.(2006). Clinical supervision and burnout: The influence of clinical supervisionfor community mental health nurses. Journal of Clinical Nursing, 15(8),1007-1015.

Ewers, P., Bradshaw, T., McGovern, J., \& Ewers, B. (2002). Does training in psychosocial interventions reduce burnout rates in forensic nurses? Journal of Advanced Nursing, 37(5), 470-476.

Hyrkas, K. (2005). Clinical supervision, burnout and job satisfaction among mental health and psychiatric nurses in Finland. Issues in Mental Health Nursing, 26(5), 531-556. 
Jenkins, R., \& Elliott, P. (2004). Stressors, burnout and social support: Nurses inacute mental health settings. Journal of Advanced Nursing, 48(6), 622-631.

Mental Health Council of Australia. (2005). Mental Health Council of Australia'ssubmission to the productivity commission health workforce study. Retrieved August 10, 2007, from http://www.pc.gov.au/study/healthworkforce/subs/sub162.pdf

Tully, A. (2004). Stress, sources of stress and ways of coping among psychiatric nursing students. Journal of Psychiatric \& Mental Health Nursing, 11(1), 43-7.

White, E., \& Roche, M. (2006). A selective review of mental health nursing in NewSouth Wales, Australia, in relation to clinical supervision. International Journal of Mental Health Nursing, 15(3), 209-219. 21574 